\title{
Knowledge of Pharmacists about Pharmacy Infection Control in Saudi Arabia
}

\section{Yousef Ahmed Alomi*, iD BSC. Pharm, MSc. Clin Pharm, BCPS, BCNSP, DiBA, CDE, Critical Care Clinical Pharmacists, TPN Clinical Pharmacist, Freelancer Business Planner, Content Editor, and Data Analyst, Riyadh, SAUDI ARABIA. \\ Ghudair Tashan Alanazi, BSc} Pharm, Pharm.D, MSc. Clin Pharm, Diploma of Epid. Critical care clinical pharmacist, Internal medicine clinical pharmacist MOH, Hafrbatin, SAUDI ARABIA.

\section{Amani Abdullah Bahdailah,} BSc. Pharm, Pharm.D, MSc. Clin Pharm Pharmaceutical Care Services, King Abdullah Bin Abdulaziz University Hospital, Riyadh, SAUDI ARABIA.

Hussa Mubarak Muwainea, Prince Sultan Military Medical City, Riyadh, SAUDI ARABIA.

Razan Alshehri, College of Pharmacy, Taif University, Tail, SAUDI ARABIA.

\section{Correspondence:}

Dr. Yousef Ahmed Alomi, Bsc. Pharm, Msc. Clin pharm, BCPS, BCNSP, DiBA, CDE Critical Care Clinical Pharmacists, TPN Clinical Pharmacist, Freelancer Business Planner, Content Editor and Data Analyst, P.O.BOX 100, Riyadh 11392, Riyadh, SAUDI ARABIA.

Phone no: +966 504417712

E-mail:yalomi@gmail.com
Copyright: @ the author(s), publisher and licensee International Journal of Pharmacology and Clinical Sciences. This is an open-access article distributed under the terms of the Creative Commons

Attribution Non-Commercial License, which permits unrestricted non-commercial use, distribution, and reproduction in any medium, provided the original work is properly cited.

This is an open access article distributed under the terms of the Creative Commons AttributionNonCommercial-ShareAlike 4.0 License

\section{Access this article online}

\begin{tabular}{|l|c|}
\hline \\
\hline
\end{tabular}

\begin{abstract}
Objectives: In this study, we aimed to assess pharmacists' knowledge about pharmacy infection control in the Kingdom of Saudi Arabia. Methods: This crosssectional survey study was conducted to assess pharmacists' knowledge about pharmacy infection control in Saudi Arabia. We used a self-reported electronic survey questionnaire and distributed it to pharmacists, including dentists from interns to consultants and pharmacy specialists in Saudi Arabia. The survey collected demographic information of the responders and their knowledge regarding some of the selected pharmacy infection control elements in dental care. We also collected information regarding the resources they use to obtain knowledge of pharmacy infection control. We used 5-point Likert response scale system with close-ended questions to obtain their responses. The data were collected through the Survey Monkey system and analyzed using Statistical Package of Social Sciences (SPSS), Jeffery's Amazing Statistics Program (JASP), and Microsoft Excel (version 16) software. Results: A total of 435 pharmacists responded to the questionnaire, with one-quarter of them coming from the central region (97 $(22.35 \%))$, and northern region (92 $(21.2 \%))$, with non-statistically significant differences between regions $(p=0.637)$. Of the total responders, 212 (48.96\%) were female, and 221 (51.04\%) were male responders, with non-statistically significant differences between them $(p=0.665)$. Most of the responders were in the age group of 24-30 years (151 (34.87\%)) and 36-40 years $(101(23.33 \%))$, with statistically significant differences between them $(p=0.000)$. The total average score of pharmacy infection control assessment for basic knowledge was 3.34, with high scores obtained for the elements knowledge of hand hygiene (3.86) and personal protective equipment (3.83). In comparison, the lowest scores were obtained for the elements knowledge of the accidental sharp devices immunization system (2.80), and the infection control pharmacist gets more salary than regular pharmacist (2.91). The total average score of knowledge of frequently used disinfection and antiseptics products at your healthcare institution (3.60) and the high scores were obtained for the elements knowledge of peroxygens (hydrogen peroxide) (4.63) and alcohol (isopropyl alcohol 70\%) (4.63). In comparison, the lowest scores were obtained for the element knowledge of phenolics (ortho-benzyl-parachlorophenol) (2.76) and the iodophors (povidone-iodine ointment 10\%) (2.99). The score for the single-test reliability analysis of McDonald's $\omega$ was 0.927 , Cronbach's awas 0.924, Gutmann's $\lambda 2$ was 0.933, Gutmann's $\lambda 6$ was 0.973, and Greater Lower Bound was 0.989. Conclusion: Pharmacists' knowledge about pharmacy infection control was inadequate in the Kingdom of Saudi Arabia. Therefore, targeting to provide the necessary education and training for undergraduate and postgraduate students to improve patient infection control within pharmaceutical care in Saudi Arabia.

Key words: Knowledge, Pharmacist, Pharmacy, Infection Control, Saudi Arabia.
\end{abstract}

\section{INTRODUCTION}

The pharmacy department consists of several sections, including inpatient pharmacy, outpatient pharmacy, and ambulatory care unit in addition to the intravenous admixture unit, the extemporaneous preparation or compounding unit, drug information section, and clinical pharmacy section. ${ }^{[1]}$ Daily, the pharmacist is involved in various activities, such as procurement, storage of medications, preparation, drug distribution, and dispensing of medication. ${ }^{[2]}$ Furthermore, they are involved in medication monitoring, preparing interventions and physician order suggestions, drug information consultation, and independent prescribing. ${ }^{[2,3]}$ All previous pharmaceutical care activities needed to be contacted and delivered to healthcare providers, pharmacy staff, and patients. The communication with pharmacy clients was either direct face-to-face contact or via online mode. Those previous contact experiences need clean or sterile weather to prevent pharmacy customers from disseminating microbial bugs or viruses. Various studies have reported mistakes committed by pharmacists in preventing transmission of infection to their patients, which have inadvertently led to the death of the patient. ${ }^{[4-16]}$ Therefore, various international pharmacy organizations such as the United States Pharmacopeia and the American Society of Health-System Pharmacists have set up regulations for infection and biohazard prevention of medications. ${ }^{[17-20]}$ Each 
medication class has different regulations and standards of preparation, such as regular intravenous medications, chemotherapy, and radiopharmaceutical medications. ${ }^{[17-20]}$ Therefore, pharmacists must have knowledge of infection, prevention, and control in a pharmacy setting. So far, various studies have been conducted to assess infection control and physician's or nurse's knowledge of infection control. ${ }^{[21-25]}$ However, to the best of our knowledge, no studies are conducted to assess pharmacists' knowledge about pharmacyrelated infection control activities. ${ }^{[26,27]}$ Therefore, in this study, we aimed to declare pharmacists' knowledge about the prevention and control of pharmacy infection in the Kingdom of Saudi Arabia.

\section{METHODS}

It was a 6-month cross-sectional study conducted to assess pharmacist's knowledge of pharmacy infection control in Saudi Arabia. We used a self-reported electronic survey questionnaire and distributed it to pharmacists from interns to consultants and specialists in Saudi Arabia. All non-pharmacists, students, and incomplete surveys were excluded from this study. The survey collected demographic information of the responders and their knowledge of selected pharmacy infection control elements in medical care, such as knowledge of frequently used disinfection and antiseptics products used at their institution. In addition, the resources of knowledge of pharmacy infection control elements in medical care were analyzed. We used 5-point Likert response scale system with close-ended questions to obtain responses. According to the previous literature with unlimited population size, in this study, the sample size was calculated for a cross-sectional study model, with the following parameters: the confidence level was $95 \%$, with a $z$ score of 1.96 and margin of error of 5-6.5\%, the population percentage of $50 \%$, and drop-out rate of $10 \%$. As a result, the sample size was calculated as 418 with a power of study of $80 \%{ }^{[28-30]}$ The response rate required for the estimated sample size was at least $60-70 \% \cdot{ }^{[30,31]}$ The survey was distributed through social media such as WhatsApp and Telegram and direct face-to-face contact. A reminder message was sent once every 1-2 weeks. Expert reviewers and pilot testing validated the survey data. Moreover, reliability tests such as McDonald's $\omega$, Cronbach's $\alpha$, Gutmann's $\lambda 2$, and Gutmann's $\lambda 6$ were calculated. The data were collected through the Survey Monkey system and analyzed by using Microsoft Excel (version 16), Statistical Package of Social Sciences (SPSS), and Jeffery's Amazing Statistics Program
(JASP) software. We conducted descriptive and frequency analysis, the goodness of fit analysis, correlation analysis, and inferential analysis on factors affecting pharmacists' knowledge about pharmacy infection control. The STROBE (Strengthening the reporting of observational studies in epidemiology statement: guidelines for reporting observational studies) guided the reporting of this study. ${ }^{[32-34]}$

\section{RESULTS}

A total of 435 pharmacists responded to the questionnaire, with one-quarter of them coming from the central region (97 (22.35\%)) and northern region (92 $(21.2 \%)$ ), with nonstatistically significant differences between the areas $(p=0.637)$. Of them, most of the responders were from a community pharmacy (81 (18.62\%)), Ministry of Health (MOH) hospitals $(69(15.86 \%))$, and military hospitals (49 $(11.26 \%)$ ), with statistically significant differences between them $(p=0.000)$. Of the total responders, 212 (48.96\%) were female, and 221 (51.04\%) were male responders, with non-statistically significant differences between them $(p=0.665)$. Most of the responders were in the age group of $24-30$ years (151 (34.87\%)) and $36-40$ years $(101(23.33 \%))$, with statistically significant differences between all age groups $(p=0.000)$. Most of the pharmacists were pharmacy staff (192 (44.55\%)) and supervisors (104 (24.13\%)), with statistical significance between them $(p=0.000)$. The majority of responders held Bachelor in Pharmacy degree (281 (64.75\%)), Master in Pharmacy degree (94 (21.66\%)), and Pharm D (90 (20.74\%)). Most of the pharmacists had work experience of 7-9 years (117 (27.08\%)) and 4-6 years (116 $(26.85 \%))$, with statistically significant between experience level $(p=0.000)$. Almost onefifth of the pharmacists practiced in clinical pharmacy (62 (18.08\%)), outpatient pharmacy (61 (17.78\%)), and inpatient pharmacy (555 $(16.03 \%))$, with statistically significant between all sites of pharmacy practice $(p=0.000)$. There is a strong positive correlation between age (years) and years of experience at the pharmacy center based on Kendall's tau_b (0.576) or Spearman's rho (0.701) correlation, with statistically significant differences between them $(p<0.001)$ (Tables 1 and 2).

The total average score for the element basic knowledge about pharmacy infection control was 3.34 , with high scores obtained for the elements knowledge of hand hygiene (3.86) and personal protective equipment (3.83), followed by hearing about the concept of pharmacy infection control (3.80) and disposal of and injuries from sharp objects (3.62). In comparison, the lowest scores were obtained for the element knowledge of the accidental sharp devices immunization system (2.80). The infection control pharmacist gets more salary than a regular pharmacist (2.91), followed by the element job description of an infection control pharmacist (2.92) and the resources of pharmacy infection control (2.96), with statistically significant differences between the responses $(p=0.000)$. Moreover, all the elements of basic knowledge were statistically significant between answers $(p=0.000)$ (Table 3$)$. The total average score for the element knowledge of frequently used disinfectants and antiseptic products at your healthcare institution (3.60), with high scores obtained for the element knowledge of peroxygens (hydrogen peroxide) (4.63), alcohol (isopropyl alcohol 70\%) (4.63), and glutaraldehyde (ortho-phthalaldehyde) (4.15). In comparison, the lowest scores were obtained for the elements, knowledge of phenolics (ortho-benzyl-para-chlorophenol) (2.76), iodophors (povidone-iodine ointment $10 \%)(2.99)$, and the quaternary ammonium compounds (Alkyl Dimethyl Benzyl Ammonium Chloride) (3.20), with statistically significant differences between the responses. Moreover, all answers were statistically significant $\quad(p=0.000) \quad$ (Table 4). Drug information resources (e.g., Lexicomp-drug information, Micromedex, and Epocrates) 280 (64.67\%), medical association literature/ guidelines/recommendations (227 (52.42\%)), and awareness lectures at primary healthcare center $(193(44.57 \%))$ were the majority of the resources used (Table 5). The scores for the single-test reliability analysis of McDonald's $\omega$ was 0.927, Cronbach's a was 0.924, Gutmann's $\lambda 2$ was 0.933 , Gutmann's $\lambda 6$ was 0.973 , and Greater Lower Bound was 0.989 .

\section{Factors influencing the pharmacy infection control assessment of knowledge and infection control products}

Various factors were affecting the pharmacist's knowledge of infection control and the pharmacy infection control products. We adjusted the significant values by using the independent samples Kruskal-Wallis test and the Bonferroni correction for multiple comparison tests. The various factor might influence the knowledge of infection control. We assessed 13 work-level sites for the basic knowledge of pharmacy infection control. The lowest average score was obtained for national guard hospitals (3.1295) and community pharmacy (3.0593), with statically significant differences between different sites $(p=0.000)$. Next, we assessed six separate age groups for the basic knowledge of pharmacy infection control with the lowest average score (2.9469) obtained for the age group 24-30 years, with 


\begin{tabular}{|c|c|c|c|c|c|c|c|}
\hline Locations & $\begin{array}{l}\text { Response } \\
\text { Count }\end{array}$ & $\begin{array}{l}\text { Response } \\
\text { Percent }\end{array}$ & $\begin{array}{l}p \text {-value } \\
(\mathrm{X} 2)\end{array}$ & Pharmacist's Qualifications & $\begin{array}{l}\text { Response } \\
\text { Count }\end{array}$ & $\begin{array}{c}\text { Response } \\
\text { Percent }\end{array}$ & $\begin{array}{l}p \text {-value } \\
\text { (X2) }\end{array}$ \\
\hline Central area & 97 & $22.35 \%$ & \multirow{6}{*}{0.637} & Diploma in Pharmacy & 1 & $0.23 \%$ & \\
\hline North area & 92 & $21.20 \%$ & & Bachelor in pharmacy & 281 & $64.75 \%$ & \\
\hline South area & \multirow{2}{*}{83} & \multirow{2}{*}{$19.12 \%$} & & 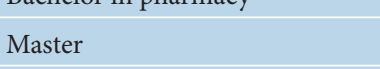 & 94 & $21.66 \%$ & \\
\hline \multirow{2}{*}{ East area } & & & & Pharm D & 90 & $20.74 \%$ & \\
\hline & 79 & $18.20 \%$ & & Ph. D & 12 & $2.76 \%$ & \\
\hline West area & 83 & $19.12 \%$ & & PGY 1 & 9 & $2.07 \%$ & \\
\hline Answered question & \multicolumn{2}{|l|}{434} & & PGY 2 & 15 & $3.46 \%$ & \\
\hline Skipped question & 1 & & & PGY 3 & 7 & $1.61 \%$ & \\
\hline Site of work & Response & Response & $p$-value & Fellowship & 4 & $0.92 \%$ & \\
\hline & Count & Percent & (X2) & Other (please specify) & 2 & $0.46 \%$ & \\
\hline MOH Hospitals & 69 & $15.86 \%$ & & Answered question & 434 & & \\
\hline Military hospitals & 49 & $11.26 \%$ & & Skipped question & 1 & & \\
\hline National Gaurd Hospital & 25 & $5.75 \%$ & & Position Held & Response & Response & \\
\hline Security forces hospitals & 41 & $9.43 \%$ & & & Count & Percent & \\
\hline KFSH\&RC & 2 & $0.46 \%$ & & Director of Pharmacy & 46 & $10.67 \%$ & \\
\hline University hospital & 24 & $5.52 \%$ & & Assistant Director of Pharmacy & 51 & $11.83 \%$ & 0.000 \\
\hline $\mathrm{MOH}$ primary care centers & 23 & $5.29 \%$ & 0.000 & Supervisor & 104 & $24.13 \%$ & \\
\hline 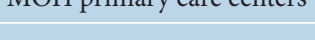 & 20 & 0.2570 & & Pharmacy staff & 192 & $44.55 \%$ & \\
\hline Private hospitals & 25 & $5.75 \%$ & & Intern & 38 & $8.82 \%$ & \\
\hline Private ambulatory care & & & & Answered question & 431 & & \\
\hline clinics & 26 & $5.98 \%$ & & Skipped question & 4 & & \\
\hline $\begin{array}{l}\text { Private primary healthcare } \\
\text { center }\end{array}$ & 26 & $5.98 \%$ & & $\begin{array}{l}\text { Years of experience at Dentists } \\
\text { career }\end{array}$ & $\begin{array}{l}\text { Response } \\
\text { Count }\end{array}$ & $\begin{array}{c}\text { Response } \\
\text { Percent }\end{array}$ & \\
\hline Community pharmacy & 81 & $18.62 \%$ & & Less than one year & 69 & $15.97 \%$ & \\
\hline Pharmaceutical companies & 38 & $8.74 \%$ & & $1-3$ & 83 & $19.21 \%$ & \\
\hline Non-employment & 3 & $0.69 \%$ & & $4-6$ & 116 & $26.85 \%$ & 0.000 \\
\hline Intern & 3 & $0.69 \%$ & & $7-9$ & 117 & $27.08 \%$ & \\
\hline Answered question & 435 & & & $10-12$ & 25 & $5.79 \%$ & \\
\hline Skipped question & $\mathbf{0}$ & & & $>12$ & 22 & $5.09 \%$ & \\
\hline Gender & Response & Response & & Answered question & 432 & & \\
\hline & Count & Percent & & Skipped question & 3 & & \\
\hline Male & 221 & $51.04 \%$ & 0.665 & Pharmacy practice & $\begin{array}{l}\text { Response } \\
\text { Count }\end{array}$ & $\begin{array}{c}\text { Response } \\
\text { Percent }\end{array}$ & \\
\hline Female & 212 & $48.96 \%$ & & Pharmacy adminstration & 1 & $0.29 \%$ & \\
\hline Answered question & 433 & & & Inpatient Pharmacy & 55 & $16.03 \%$ & \\
\hline Skipped question & 2 & & & Outpatient Pharmacy & 61 & $17.78 \%$ & \\
\hline Age & Response & Response & & Satellite Pharmacy & 17 & $4.96 \%$ & \\
\hline & Count & Percent & & Narcotics and Controlled & 22 & $6.41 \%$ & \\
\hline $24-30$ & 151 & $34.87 \%$ & & Extemporaneous Preparation & 8 & $2.33 \%$ & 0.000 \\
\hline $31-35$ & 89 & $20.55 \%$ & & Clinical Pharmacy & 62 & $18.08 \%$ & 0.000 \\
\hline $36-40$ & 101 & $23.33 \%$ & 0000 & Inventory Control & 26 & $7.58 \%$ & \\
\hline $41-45$ & 60 & $13.86 \%$ & 0.000 & Drug Information & 17 & $4.96 \%$ & \\
\hline $46-50$ & 30 & $6.93 \%$ & & IV admixture & 19 & $5.54 \%$ & \\
\hline$>50$ & 2 & $0.46 \%$ & & Community pharmacy & 33 & $9.62 \%$ & \\
\hline Answered question & 433 & & & Answered question & 343 & $6.41 \%$ & \\
\hline Skipped question & 2 & & & Skipped question & 92 & & \\
\hline
\end{tabular}




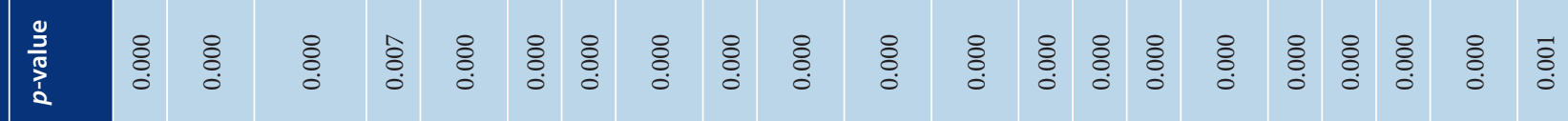

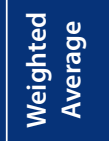

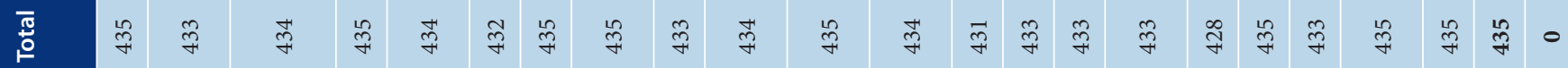

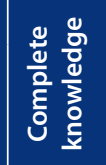

ஸ் ๘

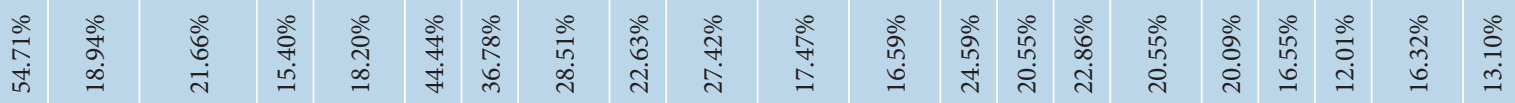

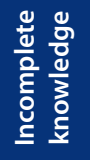

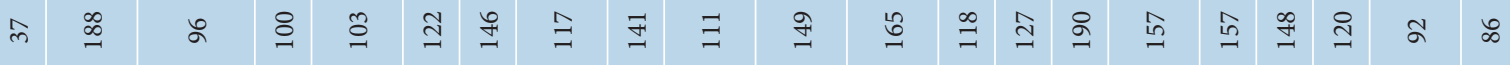

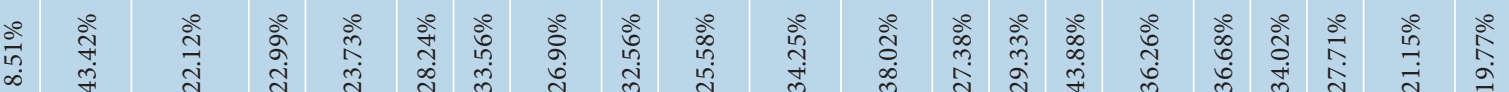

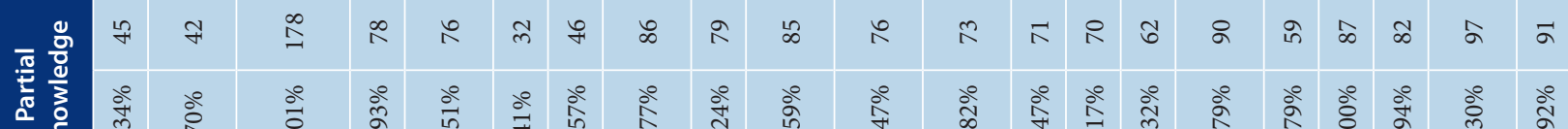

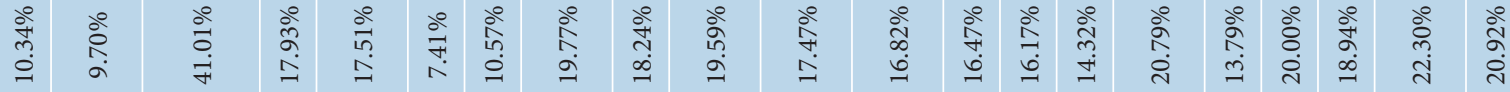

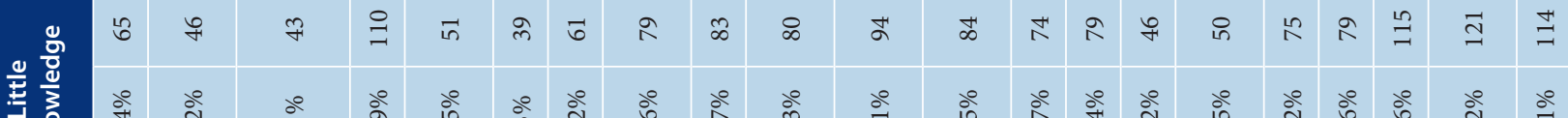

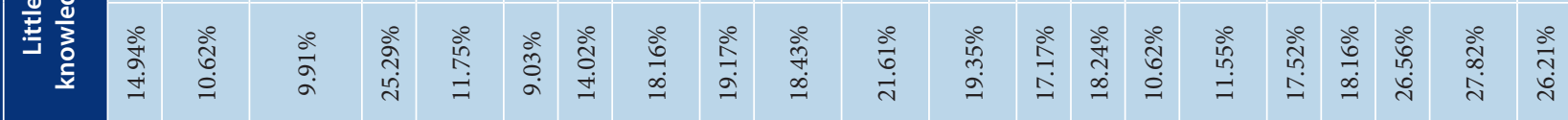

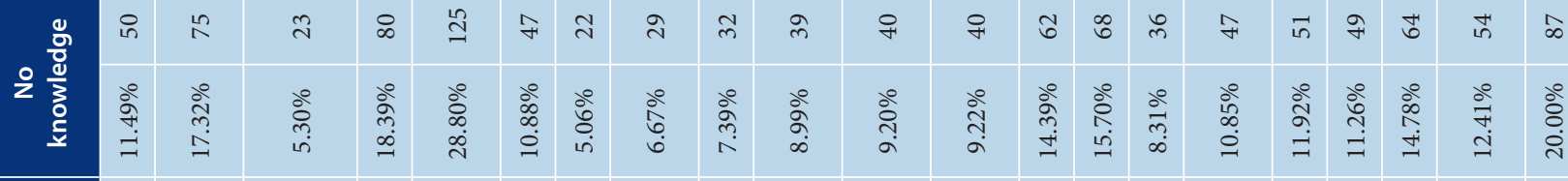

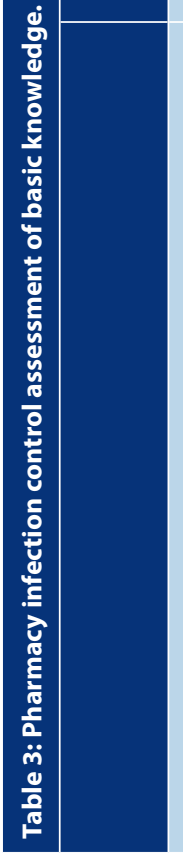

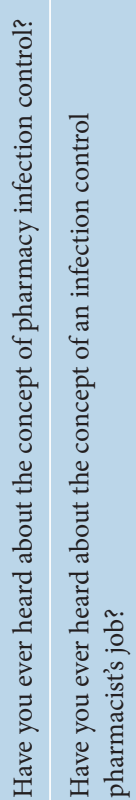

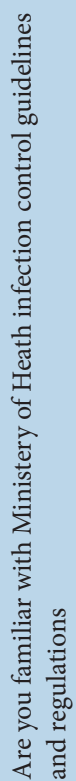
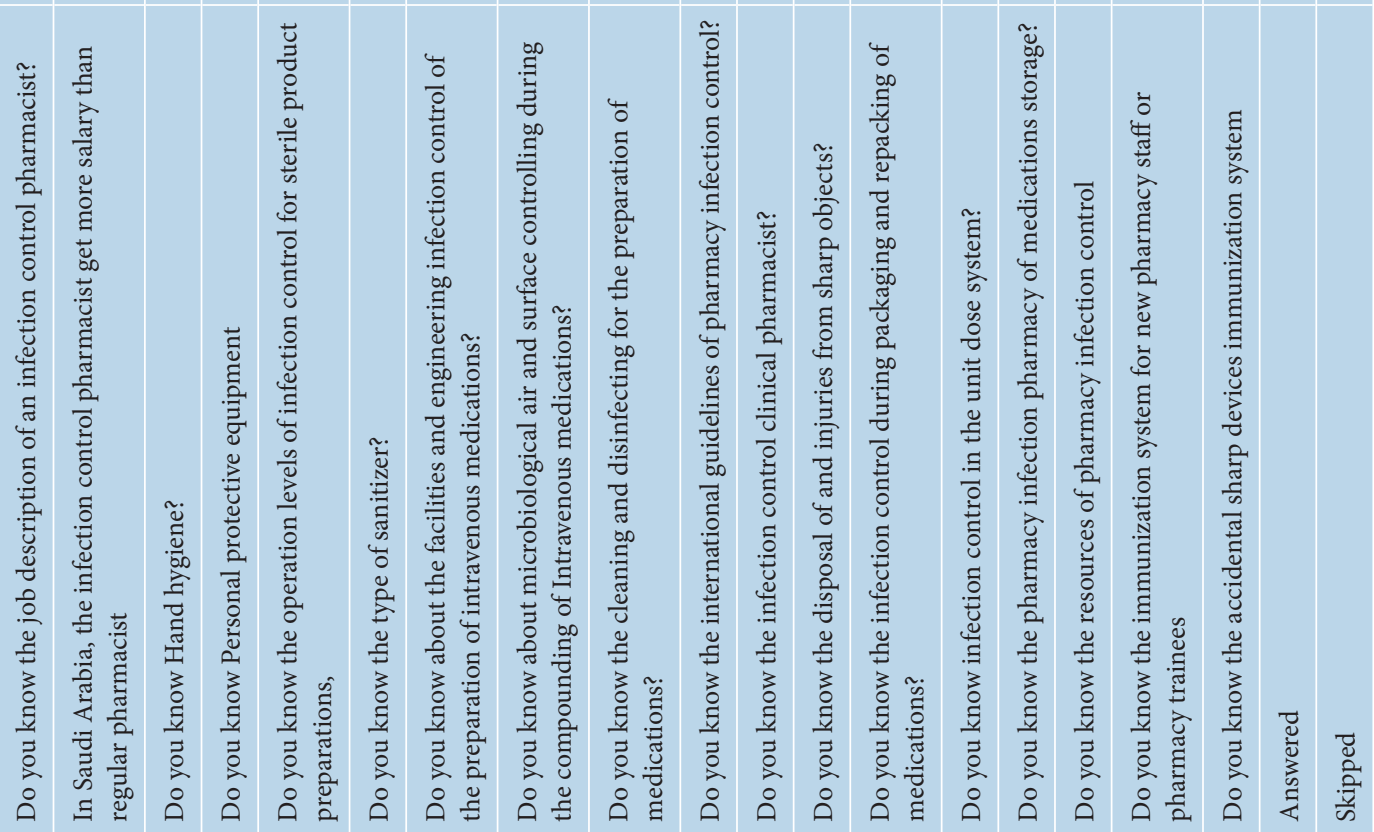


\begin{tabular}{|c|c|c|c|c|c|c|c|c|c|c|c|c|c|c|c|}
\hline \multirow[b]{2}{*}{$\begin{array}{l}\text { Peroxygens } \\
\text { (Hydrogen peroxide) }\end{array}$} & \multicolumn{2}{|c|}{$\begin{array}{c}\text { I am not familiar } \\
\text { with these } \\
\text { products }\end{array}$} & \multicolumn{2}{|c|}{ Never } & \multicolumn{2}{|c|}{ Rare } & \multicolumn{2}{|c|}{ Sometime } & \multicolumn{2}{|c|}{$\begin{array}{l}\text { Most of the } \\
\text { time }\end{array}$} & \multicolumn{2}{|c|}{ Always } & \multirow{2}{*}{$\begin{array}{c}\text { Total } \\
432 \\
\end{array}$} & \multirow{2}{*}{$\begin{array}{c}\text { Weighted } \\
\text { Average } \\
4.63\end{array}$} & \multirow{2}{*}{$\begin{array}{c}\boldsymbol{p} \text {-value } \\
0.000\end{array}$} \\
\hline & $10.88 \%$ & 47 & $8.56 \%$ & 37 & $4.40 \%$ & 19 & $12.73 \%$ & 55 & $9.49 \%$ & 41 & $53.94 \%$ & 233 & & & \\
\hline $\begin{array}{l}\text { Glutaraldehydes } \\
\text { (Ortho-phthalaldehyde) }\end{array}$ & $17.32 \%$ & 75 & $9.70 \%$ & 42 & $3.00 \%$ & 13 & $12.47 \%$ & 54 & $25.40 \%$ & 110 & $32.10 \%$ & 139 & 433 & 4.15 & 0.000 \\
\hline $\begin{array}{l}\text { Peroxygens } \\
\text { (Peracetic acid) }\end{array}$ & $18.65 \%$ & 80 & $6.06 \%$ & 26 & $6.06 \%$ & 26 & $14.22 \%$ & 61 & $43.36 \%$ & 186 & $11.66 \%$ & 50 & 429 & 3.93 & 0.000 \\
\hline Chlorhexidine gluconate & $11.09 \%$ & 48 & $7.85 \%$ & 34 & $15.01 \%$ & 65 & $16.17 \%$ & 70 & $41.80 \%$ & 181 & $8.08 \%$ & 35 & 433 & 3.94 & 0.000 \\
\hline $\begin{array}{l}\text { Alcohol } \\
\text { (Isopropyl Alcohol 70\%) }\end{array}$ & $2.86 \%$ & 12 & $8.10 \%$ & 34 & $5.00 \%$ & 21 & $22.38 \%$ & 94 & $30.24 \%$ & 127 & $31.43 \%$ & 132 & 420 & 4.63 & 0.000 \\
\hline $\begin{array}{l}\text { Iodophors (Povidone- } \\
\text { iodine solution topical 5\%) }\end{array}$ & $10.39 \%$ & 45 & $12.47 \%$ & 54 & $15.24 \%$ & 66 & $36.03 \%$ & 156 & $11.32 \%$ & 49 & $14.55 \%$ & 63 & 433 & 3.69 & 0.000 \\
\hline $\begin{array}{l}\text { Phenolics } \\
\text { (ortho-phenylphenoL) }\end{array}$ & $18.52 \%$ & 80 & $14.35 \%$ & 62 & $28.01 \%$ & 121 & $20.37 \%$ & 88 & $12.96 \%$ & 56 & $5.79 \%$ & 25 & 432 & 3.12 & 0.000 \\
\hline $\begin{array}{l}\text { Phenolics (ortho-benzyl- } \\
\text { para-chlorophenol) }\end{array}$ & $27.78 \%$ & 120 & $15.74 \%$ & 68 & $25.46 \%$ & 110 & $17.13 \%$ & 74 & $11.11 \%$ & 48 & $2.78 \%$ & 12 & 432 & 2.76 & 0.000 \\
\hline $\begin{array}{l}\text { Iodophors (Povidone- } \\
\text { iodine ointment 10\%) }\end{array}$ & $23.79 \%$ & 103 & $18.71 \%$ & 81 & $16.86 \%$ & 73 & $24.94 \%$ & 108 & $6.47 \%$ & 28 & $9.24 \%$ & 40 & 433 & 2.99 & 0.000 \\
\hline Alcohol (Ethyl alcohol) & $13.51 \%$ & 57 & $13.74 \%$ & 58 & $14.69 \%$ & 62 & $18.72 \%$ & 79 & $14.69 \%$ & 62 & $24.64 \%$ & 104 & 422 & 3.81 & 0.000 \\
\hline $\begin{array}{l}\text { Quaternary ammonium } \\
\text { compounds (Alkyl } \\
\text { dimethyl benzyl } \\
\text { ammonium chloride) }\end{array}$ & $21.48 \%$ & 93 & $19.86 \%$ & 86 & $12.93 \%$ & 56 & $21.94 \%$ & 95 & $10.39 \%$ & 45 & $13.39 \%$ & 58 & 433 & 3.2 & 0.000 \\
\hline $\begin{array}{l}\text { Quaternary ammonium } \\
\text { compounds (Benzyl } \\
\text { dimethyl octyl ammonium } \\
\text { chloride) }\end{array}$ & $18.48 \%$ & 80 & $20.09 \%$ & 87 & $15.47 \%$ & 67 & $21.02 \%$ & 91 & $20.32 \%$ & 88 & $4.62 \%$ & 20 & 433 & 3.18 & 0.000 \\
\hline $\begin{array}{l}\text { Quaternary ammonium } \\
\text { compounds (didecyl } \\
\text { dimethyl ammonium } \\
\text { chloride) }\end{array}$ & $19.17 \%$ & 83 & $19.40 \%$ & 84 & $12.93 \%$ & 56 & $25.17 \%$ & 109 & $11.55 \%$ & 50 & $11.78 \%$ & 51 & 433 & 3.26 & 0.000 \\
\hline $\begin{array}{l}\text { Chlorine and } \\
\text { chlorine compounds } \\
\text { (Hypochlorites) }\end{array}$ & $21.53 \%$ & 93 & $11.81 \%$ & 51 & $13.19 \%$ & 57 & $22.69 \%$ & 98 & $17.13 \%$ & 74 & $13.66 \%$ & 59 & 432 & 3.43 & 0.000 \\
\hline $\begin{array}{l}\text { Chlorine and chlorine } \\
\text { compounds (Chlorine } \\
\text { dioxide) }\end{array}$ & $19.35 \%$ & 84 & $13.82 \%$ & 60 & $12.21 \%$ & 53 & $13.82 \%$ & 60 & $28.34 \%$ & 123 & $12.44 \%$ & 54 & 434 & 3.55 & 0.000 \\
\hline $\begin{array}{l}\text { Chlorine and chlorine } \\
\text { compounds (Chloramine-t } \\
\text { trihydrate) }\end{array}$ & $21.71 \%$ & 94 & $14.78 \%$ & 64 & $10.16 \%$ & 44 & $17.32 \%$ & 75 & $26.79 \%$ & 116 & $9.24 \%$ & 40 & 433 & 3.4 & 0.000 \\
\hline Answered & & & & & & & & & & & & & & 434 & \\
\hline Skipped & & & & & & & & & & & & & & 1 & \\
\hline
\end{tabular}

statistically significant differences between different age groups $(p=0.000)$. Then, we assessed 12 practice areas for the basic knowledge of pharmacy infection control. The highest average score was obtained for the IV admixture section (4.2115), with a statistically significant difference between different practice areas $(p=0.000)$. Six levels of experience affected the basic knowledge of pharmacy infection control, with the lowest score (2.8477) obtained for the experience of less than one year, with a statistically significant difference between all levels $(p=0.000)$. Five current positions affected the basic knowledge of pharmacy infection control, with the lowest score (2.8731) obtained for interns with a statistically significant difference between all positions held $(p=0.000)$. There was a medium relationship ( $R=0.479$ with $p=0.000)$ between pharmacy infection control knowledge and its factors. Three out of seven factors did not show any significant differences $(p>0.05)$.
However, worksite explained $26.1 \%$ negative relationship, gender explained $10.7 \%$ positive relationship, practice area described $26.4 \%$ positive relationship, and current position held explained a $28 \%$ negative relationship to the variation. They were a statistically significant difference between them $(p=0.000,0.036,0.000$, and 0.000 , respectively) through the multiple regression model and confirmed by the Bootstrap model. The relationship was verified by the non-existence of multi-collinearity 


\begin{tabular}{|c|c|c|}
\hline \multirow{2}{*}{$\begin{array}{l}\text { Answer Choices } \\
\text { Health practitioners }\end{array}$} & \multicolumn{2}{|c|}{ Responses } \\
\hline & 170 & $39.26 \%$ \\
\hline Scientific literature & 131 & $30.25 \%$ \\
\hline Peer discussions & 151 & $34.87 \%$ \\
\hline Medical association literature/guidelines/recommendations & 227 & $52.42 \%$ \\
\hline Drug information resources ( Lexicomp-drug information, Micromedex, Epocrates ..etc & 280 & $64.67 \%$ \\
\hline SFDA website & 131 & $30.25 \%$ \\
\hline Drug Bulletin & 59 & $13.63 \%$ \\
\hline Relatives and friends & 72 & $16.63 \%$ \\
\hline Pharmacy infection control education courses & 132 & $30.48 \%$ \\
\hline Internet & 94 & $21.71 \%$ \\
\hline The drug information center at the hospital & 158 & $36.49 \%$ \\
\hline Awareness lectures in a hospital & 170 & $39.26 \%$ \\
\hline Awareness lectures primary healthcare center & 193 & $44.57 \%$ \\
\hline Healthcare care awareness events at the market & 69 & $15.94 \%$ \\
\hline Answered & 433 & \\
\hline Skipped & 2 & \\
\hline
\end{tabular}

between the years of experience factor with variance inflation factor $(\mathrm{VIF}=1.342,1.120$, 1.239 , and 1.410 , respectively) for less than 3 or $5^{[35-37]}$ (Table 6).

On the contrary, 5 locations affected the pharmacy infection control products with the low average scores (3.3210 and 3.3156) obtained for the central and western region, with statistically significant differences between all areas $(p=0.000)$. Next, among the 13 levels of the worksite that affected the pharmacy infection control, the lowest score (3.0564) was obtained for the pharmaceutical company, with statistically significant differences between different worksites $(p=0.000)$. Then, we tested six different age groups for their knowledge of pharmacy infection control products. The lowest average score (2.9753) was obtained for the age group 24-30 years, with statistically significant differences between different age groups $(p=0.000)$. Then, we tested 12 practice areas that affected the knowledge of pharmacy infection control products, with the highest

\begin{tabular}{|c|c|c|c|c|c|c|c|c|c|c|c|c|c|c|}
\hline & \multirow[b]{2}{*}{ Model } & \multirow[b]{2}{*}{$\mathbf{R}$} & \multirow[b]{2}{*}{$\begin{array}{c}\mathbf{R} \\
\text { Square }\end{array}$} & \multirow[b]{2}{*}{ F } & \multirow[b]{2}{*}{ Sig. } & \multicolumn{2}{|c|}{$\begin{array}{l}\text { Unstandardized } \\
\text { Coefficients }\end{array}$} & \multirow{2}{*}{$\begin{array}{c}\begin{array}{c}\text { Standardized } \\
\text { Coefficients }\end{array} \\
\text { Beta }\end{array}$} & \multirow[b]{2}{*}{$\mathbf{t}$} & \multirow[b]{2}{*}{ Sig. } & \multicolumn{2}{|c|}{$\begin{array}{l}95.0 \% \text { Confidence } \\
\text { Interval for B }\end{array}$} & \multicolumn{2}{|c|}{$\begin{array}{l}\text { Collinearity } \\
\text { Statistics }\end{array}$} \\
\hline & & & & & & B & $\begin{array}{l}\text { Std. } \\
\text { Error }\end{array}$ & & & & $\begin{array}{l}\text { Lower } \\
\text { Bound }\end{array}$ & $\begin{array}{l}\text { Upper } \\
\text { Bound }\end{array}$ & Tolerance & VIF \\
\hline \multirow[t]{8}{*}{1} & (Constant) & \multirow[t]{8}{*}{$.479^{\mathrm{b}}$} & \multirow[t]{8}{*}{.229} & \multirow[t]{8}{*}{14.164} & \multirow[t]{8}{*}{$.000^{\mathrm{b}}$} & 3.403 & 0.280 & & 12.161 & 0.000 & 2.852 & 3.953 & & \\
\hline & Location & & & & & 0.016 & 0.026 & 0.031 & 0.633 & 0.527 & -0.034 & 0.067 & 0.954 & 1.048 \\
\hline & Site of work & & & & & -0.054 & 0.012 & -0.261 & -4.680 & 0.000 & -0.077 & -0.032 & 0.745 & 1.342 \\
\hline & Age (years) & & & & & 0.041 & 0.043 & 0.070 & 0.949 & 0.343 & -0.044 & 0.125 & 0.427 & 2.340 \\
\hline & Pharmacist gender & & & & & 0.165 & 0.078 & 0.107 & 2.101 & 0.036 & 0.011 & 0.319 & 0.893 & 1.120 \\
\hline & Practice area & & & & & 0.061 & 0.012 & 0.264 & 4.925 & 0.000 & 0.037 & 0.086 & 0.807 & 1.239 \\
\hline & Current Position & & & & & -0.190 & 0.039 & -0.280 & -4.911 & 0.000 & -0.266 & -0.114 & 0.709 & 1.410 \\
\hline & $\begin{array}{l}\text { Years of } \\
\text { experiences }\end{array}$ & & & & & 0.073 & 0.040 & 0.130 & 1.837 & 0.067 & -0.005 & 0.150 & 0.461 & 2.170 \\
\hline
\end{tabular}

a. Dependent Variable: Pharmacist infection control assessment of basic knowledge, Predictors b: (Constant), Location, Site of work, Age (years), Pharmacist gender, Practice area, years of experience, and current Position.

\begin{tabular}{|c|c|c|c|c|c|c|c|}
\hline \multicolumn{8}{|c|}{ Bootstrap for Coefficients } \\
\hline & \multirow[b]{3}{*}{ Model } & \multirow[b]{3}{*}{ B } & \multicolumn{5}{|c|}{ Bootstrap $^{a}$} \\
\hline & & & \multirow[b]{2}{*}{ Bias } & \multirow[b]{2}{*}{ Std. Error } & \multirow[b]{2}{*}{ Sig. (2-tailed) } & \multicolumn{2}{|c|}{ 95\% Confidence Interval } \\
\hline & & & & & & Lower & Upper \\
\hline \multirow[t]{8}{*}{1} & (Constant) & 3.403 & 0.013 & 0.315 & 0.001 & 2.772 & 4.008 \\
\hline & Location & 0.016 & 0.000 & 0.028 & 0.588 & -0.043 & 0.068 \\
\hline & Site of work & -0.054 & 0.000 & 0.013 & 0.001 & -0.079 & -0.029 \\
\hline & Age (years) & 0.041 & -0.002 & 0.043 & 0.343 & -0.038 & 0.124 \\
\hline & Pharmacist gender & 0.165 & -0.003 & 0.082 & 0.051 & 0.001 & 0.328 \\
\hline & Practice area & 0.061 & 0.000 & 0.015 & 0.001 & 0.030 & 0.090 \\
\hline & Current Position & -0.190 & -0.002 & 0.040 & 0.001 & -0.271 & -0.116 \\
\hline & Years of experiences & 0.073 & 0.001 & 0.042 & 0.096 & -0.009 & 0.163 \\
\hline
\end{tabular}

a. Unless otherwise noted, bootstrap results are based on 1000 bootstrap samples 
average score obtained for the IV admixture section (4.3110), with statistically significant differences between all areas $(p=0.000)$. Next, we tested five current positions held that affected the knowledge of pharmacy infection control products, and the lowest score (3.0467) was obtained for interns with a statistically significant difference between positions held $(p=0.000)$. Next, six levels of years of experience affected the basic knowledge of pharmacy infection control, with the lowest score (2.8777) obtained for less than one year, with statistically significant differences between all levels of work experience $(\mathrm{p}=0.000)$. There was a medium relationship ( $R=0.433$ with $p=0.000)$ between knowledge of frequently used disinfection and antiseptics products and factors. Four out of seven factors did not show any significant differences between them $(p>0.05)$. However, age explained $18 \%$ of the positive relationship, and the current position explained $18.7 \%$ of the negative relationship. Years of experience explained $15.8 \%$ of the variation in the knowledge of frequently used disinfection and antiseptics products and factors, with a statistically significant difference $(p=0.018$, 0.002 , and 0.031 , respectively) through multiple regression model and confirmed by Bootstrap model. The relationship was verified by the non-existence of multi-collinearity with the years of experience factor with the Variance Inflation Factor $(\mathrm{VIF}=2.340,1.410$, and 2.170, respectively) less than three or 5 five ${ }^{[35-37]}$ (Table 7).

\section{DISCUSSION}

Knowledge of infection control is required in the pharmacy practice. ${ }^{[17-20]}$ All pharmacists should be aware of infection control during the preparation and dispensing of medications. ${ }^{[17-20]}$ The infection control knowledge is also necessary for the preparation of intravenous admixtures, inpatient pharmacy, and outpatient pharmacy practice sections. ${ }^{[17-20]}$ Furthermore, it is essential to prevent infection-related problems during a pandemic or regular period. Therefore, the description of pharmacist knowledge of infection control is critical. As a result, this study with convenient sampling obtained optimal sample size better than previous pharmacy studies or healthcare professional studies, ${ }^{[21-27]}$ with non-significant differences $(p>0.05)$ distribution of responder's geographic location and gender among male and female responders. On the contrary, the number of responders from the different worksites, age groups, different academic qualifications, and positions held were unequal. That's reflected the background information of a variety of types or groups of responders. Unfortunately, it cannot publish demographic data in actual practice. The average knowledge of pharmacy infection control of pharmacists was found to be inadequate, which resembled previous studies. ${ }^{[21,22,25]}$ However, our results were better than two previous studies ${ }^{[23,25]}$ and lower than another study. ${ }^{[24]}$ Most pharmacists were familiar with daily activities

\begin{tabular}{|c|c|c|c|c|c|c|c|c|c|c|c|c|c|c|}
\hline & \multirow[b]{2}{*}{ Model } & \multirow[b]{2}{*}{$\mathbf{R}$} & \multirow[b]{2}{*}{$\begin{array}{c}\mathbf{R} \\
\text { Square }\end{array}$} & \multirow[b]{2}{*}{$\mathbf{F}$} & \multirow[b]{2}{*}{ Sig. } & \multicolumn{2}{|c|}{$\begin{array}{l}\text { Unstandardized } \\
\text { Coefficients }\end{array}$} & \multirow{2}{*}{$\begin{array}{c}\begin{array}{c}\text { Standardized } \\
\text { Coefficients }\end{array} \\
\text { Beta }\end{array}$} & \multirow[b]{2}{*}{$\mathbf{t}$} & \multirow[b]{2}{*}{ Sig. } & \multicolumn{2}{|c|}{$\begin{array}{l}\mathbf{9 5 . 0} \% \text { Confidence } \\
\text { Interval for B }\end{array}$} & \multicolumn{2}{|c|}{$\begin{array}{l}\text { Collinearity } \\
\text { Statistics }\end{array}$} \\
\hline & & & & & & B & $\begin{array}{l}\text { Std. } \\
\text { Error }\end{array}$ & & & & $\begin{array}{l}\text { Lower } \\
\text { Bound }\end{array}$ & $\begin{array}{l}\text { Upper } \\
\text { Bound }\end{array}$ & Tolerance & VIF \\
\hline \multirow[t]{8}{*}{1} & (Constant) & \multirow[t]{8}{*}{$.433^{\mathrm{b}}$} & \multirow[t]{8}{*}{.188} & \multirow[t]{8}{*}{10.991} & \multirow[t]{8}{*}{$.000^{\mathrm{b}}$} & 3.308 & 0.364 & & 9.087 & 0.000 & 2.592 & 4.024 & & \\
\hline & Location & & & & & -0.003 & 0.033 & -0.005 & -0.104 & 0.917 & -0.069 & 0.062 & 0.954 & 1.048 \\
\hline & Site of work & & & & & -0.029 & 0.015 & -0.109 & -1.904 & 0.058 & -0.059 & 0.001 & 0.745 & 1.342 \\
\hline & Age (years) & & & & & 0.133 & 0.056 & 0.180 & 2.379 & 0.018 & 0.023 & 0.242 & 0.427 & 2.340 \\
\hline & Pharmacist gender & & & & & 0.129 & 0.102 & 0.066 & 1.264 & 0.207 & -0.072 & 0.329 & 0.893 & 1.120 \\
\hline & Practice area & & & & & 0.028 & 0.016 & 0.095 & 1.721 & 0.086 & -0.004 & 0.060 & 0.807 & 1.239 \\
\hline & Current Position & & & & & -0.161 & 0.050 & -0.187 & -3.194 & 0.002 & -0.260 & -0.062 & 0.709 & 1.410 \\
\hline & $\begin{array}{l}\text { Years of } \\
\text { experiences }\end{array}$ & & & & & 0.111 & 0.051 & 0.158 & 2.165 & 0.031 & 0.010 & 0.213 & 0.461 & 2.170 \\
\hline
\end{tabular}

a. Dependent Variable: the Knowledge and frequency used the disinfection and antiseptics products, Predictors b: (Constant), Location, Site of work, Age (years), Pharmacist gender, Practice area, years of experience, and current Position

\begin{tabular}{|c|c|c|c|c|c|c|c|}
\hline \multicolumn{8}{|c|}{ Bootstrap for Coefficients } \\
\hline & \multirow[b]{3}{*}{ Model } & \multirow[b]{3}{*}{ B } & \multicolumn{5}{|c|}{ Bootstrap ${ }^{a}$} \\
\hline & & & \multirow[b]{2}{*}{ Bias } & \multirow[b]{2}{*}{ Std. Error } & \multirow[b]{2}{*}{ Sig. (2-tailed) } & \multicolumn{2}{|c|}{ 95\% Confidence Interval } \\
\hline & & & & & & Lower & Upper \\
\hline \multirow[t]{8}{*}{1} & (Constant) & 3.308 & -0.014 & 0.393 & 0.001 & 2.543 & 4.058 \\
\hline & Location & -0.003 & -0.001 & 0.037 & 0.909 & -0.077 & 0.065 \\
\hline & Site of work & -0.029 & 0.001 & 0.016 & 0.073 & -0.059 & 0.004 \\
\hline & Age (years) & 0.133 & $6.559 \mathrm{E}-05$ & 0.050 & 0.008 & 0.041 & 0.239 \\
\hline & Pharmacist gender & 0.129 & -0.002 & 0.105 & 0.235 & -0.078 & 0.331 \\
\hline & Practice area & 0.028 & 0.001 & 0.020 & 0.174 & -0.011 & 0.064 \\
\hline & Current Position & -0.161 & 0.004 & 0.046 & 0.001 & -0.245 & -0.066 \\
\hline & Years of experiences & 0.111 & 0.000 & 0.051 & 0.026 & 0.010 & 0.210 \\
\hline
\end{tabular}

a. Unless otherwise noted, bootstrap results are based on 1000 bootstrap samples 
such as hand hygiene and personal protective equipment (PPE), ${ }^{[24]}$ in addition to the concept of pharmacy infection control. This result is expected because some of the elements mentioned above are daily necessary practice. In contrast, pharmacists did not know some aspects such as immunization of accidental sharp devices and pharmacy job descriptions. Besides, the pharmacist infection control got additional salary and references used for pharmacy infection control. ${ }^{[22]}$ Thus, we can say that there was no foundation for pharmacy infection control, or there were no pharmacists assigned for infection control jobs in pharmacy services.

In this study, the pharmacist had insufficient knowledge of infection control compounds. However, they were familiar with some materials such as hydrogen peroxide. The pharmacist dispenses the medications for oral dental disease. In comparison, Isopropyl alcohol was dispensed for intravenous admixture cleaning the laminar flow hood before and after sterile preparation. Besides, Gluteruldehyde is used for hard places cleaning in the pharmacy department. Some infection control products are not widely used in pharmacies, such as phenols products used for toilet cleaning and Indiofore for surgical cleaning or soft places cleaning. Also, other products not commonly used in pharmacy practice, such as quaternary ammonium compound for clothes washing laundry. However, these compounds should be familiarized with by pharmacists as they distribute them. Drug information resources and medical guidelines were the majority of the references which a pharmacist goes through.

\section{Factors affecting the knowledge of pharmacists about pharmacy infection control and related products}

Various factors might affect the knowledge of pharmacy infection control. For example, the worksite affected the knowledge of infection control, such as community pharmacy and non-MOH governmental sites. The finding showed that there is an inadequacy of awareness or education and training about pharmacy infection control. Additional factors affected the knowledge assessment of pharmacy infection control, such as young age, lower positive and few years of experiences, which agrees with the results of a previous study. ${ }^{[21,24,25]}$ That was related to the responders needed some time to become familiar with pharmacy infection control procedures. However, the practice area such as the Intravenous admixture section showed higher knowledge of infection control because they deal with it daily. Two factors positively affected the knowledge of infection control, such as gender and practice area. The female responders practiced more accurate infection control methods than male responders, which might expand their knowledge about pharmacy infection control, different from the previous study. ${ }^{[22]}$ Regression analysis confirmed it as a positive dependent factor; the pharmacy infection control knowledge increased with gender. The other two negative depending factors with negative proportion were the site of work and position held. That might be related to the practice and experiences of healthcare organizations. The knowledge of pharmacy infection was decreasing due to inadequate infection control practice by the healthcare workers. If the responders get higher positions or jobs, they will forget the pharmacy infection knowledge because they did not practice and were busy with the administration issues.

On the contrary, some factors might positively affect the knowledge of infection control products, such as location, central and western region. The pharmacist is not dealing with or disturbing the infection control products. The infection control department receives and distributes the products to all medical and non-medical sections at healthcare institutions. Additional factors such as young age, less experience, low position held, and less understanding of pharmacy infection control might affect the application of this knowledge, which agrees with the results of previous studies ${ }^{[21,24,25]}$ and contrary to the other. ${ }^{[23]}$ However, the intravenous admixture practice site showed high knowledge of infection control material. That was possible because they use the products daily. Two factors might have a positive effect on the knowledge, such as age and years of experience. However, with the higher position held, the knowledge of infection control products was found to be reduced because they do not deal with the infection control products on a daily basis.

\section{Limitations}

In this study, we aimed to assess the knowledge of pharmacists about infection control. As a result, we obtained some good results, such as the study having a good sample size and a highreliability survey. Moreover, it had comparable demographic data based on region and gender. However, there were some limitations to this study. First, the demographic data was unequal such as age, years of experience, and current position held. In addition, there were only a few studies to compare with the results of this study. Therefore, further studies are required with equal demographic information and regular or periodic survey about knowledge pharmacy infection control in Saudi Arabia.

\section{CONCLUSION}

In summary, the knowledge of pharmacists about pharmacy infection control was found to be insufficient. The results were affected by various factors, such as young age, less work experience, lower position held, had inadequate knowledge. Therefore, education and training should be provided during the residency program to increase their knowledge about infection control. Consequently, we recommend that pharmacy infection control be founded at all healthcare organizations in Saudi Arabia.

\section{ACKNOWLEDGEMENT}

None.

\section{CONFLICT OF INTEREST}

The authors declare that there is no conflict of interest.

\section{Funding}

None

\section{Consent for Publications}

Informed consent was obtained from all the participants

\section{Ethical Approval}

This research is exempted from research and ethical committee or an institutional review board (IRB) approval.

https://www.hhs.gov/ohrp/regulations-andpolicy/decision-charts-2018/index.html

\section{ABBREVIATIONS}

MOH: Ministry of Health; KSA: Kingdom of Saudi Arabia; SPSS: Statistical package of social sciences; JASP: Jeffery's Amazing Statistics Program; STROBE: Strengthening the reporting of observational studies in epidemiology; VIF: Variance Inflation Factor; KFSHRC: King Faisal Specialist Hospital and Research Center.

\section{ORCID ID}

Yousef Ahmed Alomi (iD https://orcid. org/0000-0003-1381-628X

\section{REFERENCES}

1. Settings $P$ [ASHP guidelines]. Minimum standard for pharmacies in hospitals. Am J Heal Pharm. Vol. 52(23); 1995;2711-7.

2. ASHP guidelines on hospital drug distribution and control (with references). Am J Hosp Pharm. 1980;37(8):1097-103. PMID 7405941.

3. American Society of Health System Pharmacists. ASHP guidelines on a standardized method for pharmaceutical care. American Journal of HealthSystem Pharmacy. 1996;53(14):1713-6. doi: 10.1093/ajhp/53.14.1713. 
4. Shehab N, Brown MN, Kallen AJ, Perz JF. US Compounding Pharmacy-Related Outbreaks, 2001-2013: Public Health and Patient Safety Lessons Learned. J Patient Saf. 2018;14(3):16473. doi: 10.1097/PTS.0000000000000188. PMID 26001553.

5. Staes C, Jacobs J, Mayer J, Allen J. Description of outbreaks of health-care-associated infections related to compounding pharmacies, 2000-12. Am J Health Syst Pharm. 2013;70(15):1301-12. doi: 10.2146/ajhp130049, PMID 23867487.

6. Centers for Disease Control and Prevention (CDC). CDC grand rounds: Preventing unsafe injection practices in the US health-care system. MMWR Morb Mortal Wkly Rep. 2013;62(21):423-5. PMID 23718950

7. Centers for Disease Control and Prevention (CDC). Multistate outbreak of fungal infection associated with injection of methylprednisolone acetate solution from a single compounding pharmacy United States, 2012. MMWR Morb Mortal Wkly Rep. 2012;61(41):839-42. PMID 23076093

8. Yablon BR, Dantes R, Tsai V, Lim R, MoultonMeissner $H$, Arduino $M$, et al. Outbreak of Pantoea agglomerans Bloodstream Infections at an Oncology Clinic-Illinois, 2012-2013. Infect Control Hosp Epidemiol. 2017;38(3):314-9. doi: 10.1017/ice.2016.265, PMID 27919308.

9. Wiese $A D$, Griffin MR, Schaffner W, Stein CM, Greevy RA, Mitchel EF, et al. Opioid Analgesic use and Risk for Invasive Pneumococcal Diseases: A Nested Case-Control Study. Ann Intern Med. 2018;168(6):396-404. doi: 10.7326/M17-1907, PMID 29435555.

10. Souza Dias MB, Cavassin LGT, Stempliuk V, Xavier LS, Lobo RD, Sampaio JLM, et al. Multiinstitutional outbreak of Burkholderia cepacia complex associated with contaminated mannitol solution prepared in compounding pharmacy. Am J Infect Control. 2013:41(11):1038-42. doi: 10.1016/j.ajic.2013.01.033, PMID 23663863.

11. Kainer MA, Reagan DR, Nguyen DB, Wiese $A D$, Wise ME, Ward J, et al. Tennessee Fungal Meningitis Investigation Team. Fungal infections associated with contaminated methylprednisolone in Tennessee. N Engl J Med. 2012;367(23):2194-203. doi: 10.1056/NEJMoa1212972, PMID 23131029.

12. Gershman MD, Kennedy DJ, Noble-Wang J, Kim C, Gullion J, Kacica M, et al. Pseudomonas fluorescens Investigation Team. Multistate outbreak of Pseudomonas fluorescens bloodstream infection after exposure to contaminated heparinized saline flush prepared by a compounding pharmacy. Clin Infect Dis. 2008;47(11):1372-9. doi: 10.1086/592968, PMID 18937575

13. Selenic D, Dodson DR, Jensen B, Arduino MJ, Panlilio A Archibald LK. Enterobacter cloacae bloodstream infections in pediatric patients traced to a hospital pharmacy. Am J Health Syst Pharm. 2003;60(14):1440-6. doi: 10.1093/ajhp/60.14.1440. PMID 12892028

14. Held MR, Begier EM, Beardsley DS, Browne FA, Martinello RA, Baltimore RS, et al. Lifethreatening sepsis caused by Burkholderia cepacia from contaminated intravenous flush solutions prepared by a compounding pharmacy in another state. Pediatrics. 2006:118(1):e212-5. doi: 10.1542/peds.20052617, PMID 16785290.

15. Stucki C, Sautter AM, Favet J, Bonnabry P. Microbial contamination of syringes during preparation: the direct influence of environmenta cleanliness and risk manipulations on endproduct quality. Am J Health Syst Pharm. 2009;66(22):2032-6. doi: 10.2146/ajhp070681 PMID 19890087.

16. Opilla M. Epidemiology of bloodstream infection associated with parenteral nutrition. Am J Infect Control. 2008:36(10):S173.e5-8. doi: 10.1016/1. ajic.2008.10.007, PMID 19084152.

17. Kastango ES, American Society of HealthSystem Pharmacists (ASHP). Blueprint for implementing USP chapter 797 for compounding sterile preparations. Am J Health Syst Pharm. 2005:62(12):1271-88. doi: 10.1093/ajhp/62.12.1271 PMID 15947127

18. United States Pharmacopeia, General Chapter USP <800> Hazardous Drugs- Handling in Healthcare Settings. The United States pharmacopeial convention. 2017;1-20.

19. USP, General Chapter USP < 795> Pharmaceutical Compounding - Nonsterile Preparations. USP 42NF [internet]. p. 1-13; 2019. Available from: http:// www.usp.org/compounding/general-chapter-795 [cited 24/11/2021]. In:

20. United States Pharmacopia. USP General Chapter $<825>$ radiopharmaceuticals - preparation, compounding, dispensing, and repackaging United States Pharmacopia. 2019:1-29://www.

21. Kim KM, Choi JS. Self-perceived competency of infection control nurses based on Benner's framework: A nationwide survey in Korea. Appl Nurs Res. 2015;28(2):175-9. doi: 10.1016/j apnr.2014.09.010, PMID 25315139.

22. Khubrani A, Albesher M, Alkahtani A, Alamri F, Alshamrani M, Masuadi E. Knowledge and information sources on standard precautions and infection control of health sciences students at King Saud Bin Abdulaziz University for Health Sciences, Saudi Arabia, Riyadh. J Infect Public Health. 201;11(4):546-9. doi: 10.1016/j. jiph.2017.10.013, PMID 29137958.

23. Peres D, Severo M, Ferreira MA. Knowledge, source of information, and perception of Portuguese medical students and junior doctors of infection control precautions. Am J Infect Control. 2016;44(12):1723-5. doi: 10.1016/j. ajic.2016.05.018, PMID 27499194.

24. Adegboye MB, Zakari S, Ahmed BA, Olufemi GH. Knowledge, awareness and practice of infection control by health care workers in the intensive care units of a tertiary hospital in Nigeria. Af Health Sci. 2018;18(1):72-8. doi: 10.4314/ahs. v18i1.11, PMID 29977260

25. Alhumaid S, Al Mutair A, Al Alawi Z, Alsuliman M, Ahmed GY, Rabaan AA, et al. Kknowledge of infection prevention and control among healthcare workers and factors influencing compliance: A systematic review. Antimicrob Resist Infect
Control. 2021;10(1):86. doi: 10.1186/s13756-02100957-0, PMID 34082822.

26. Murtough SM, Hiom SJ, Palmer M, Russell AD A survey of rotational use of biocides in hospital pharmacy aseptic units. J Hosp Infect. 2002;50(3):228-31. doi: 10.1053/jhin.2001.1155, PMID 11886201.

27. Sum ZZ, Ow CJW. Community pharmacy response to infection control during COVID-19. A cross-sectional survey. Res Social Adm Pharm. 2021;17(1):1845-52. doi: 10.1016/j. sapharm.2020.06.014, PMID 33317763

28. Charan J, Biswas T. How to calculate sample size for different study designs in medical research? Indian J Psychol Med. 2013;35(2):121-6. doi: 10.4103/0253-7176.116232, PMID 24049221.

29. Pourhoseingholi MA, Vahedi M, Rahimzadeh M Sample size calculation in medical studies Gastroenterol Hepatol Bed Bench. 2013:6(1):14-7. PMID 24834239

30. Ezhumalai DG. How Big A Sample Do I Require? Annals of SBV. 2017;6(1):39-41. doi: 10.5005/jpjournals-10085-6113.

31. Johnson TP, Wislar JS. Response rates and nonresponse errors in surveys [internet] JAMA. 2012:307(17):1805-6. doi: 10.1001/ jama.2012.3532, PMID 22550194.

32. Von Elm E, Altman DG, Egger M, Pocock SJ, Gøtzsche PC, Vandenbroucke JP. The strengthening the reporting of observational studies in epidemiology (STROBE) statement: Guidelines for reporting observational studies. PLOS Med. 2007;4(10):1623-7. doi: 10.1371/ journal.pmed.0040296.

33. Von Elm E, Altman DG, Egger $M$, Pocock SJ, Gøtzsche PC, Vandenbroucke JP. The Strengthening the Reporting of Observational Studies in Epidemiology (STROBE) statement: guidelines for reporting observational studies [internet]. Vol. 370; 2007. Available from: http:// www.thelancet.com. Available from: http://www. plosmedicine.org [cited 24/11/2021].

34. Langan SM, Schmidt SA, Wing $K$, Ehrenstein $V$ Nicholls SG, Filion KB, et al. The reporting of studies conducted using observational routinely collected health data statement for pharmacoepidemiology (RECORD-PE). BMJ 2018;363:k3532. doi: 10.1136/bmj.k3532, PMID 30429167.

35. Liao D, Valliant R. Variance inflation factors in the analysis of complex survey data. Surv Methodol. 2012;38(1):53-62

36. Akinwande MO, Dikko HG, Samson A. Variance inflation Factor: As a condition for the inclusion of suppressor variable(s) in regression analysis. Open J Stat. 2015:05(7):754-67. doi: 10.4236/ ojs.2015.57075.

37. Thompson CG, Kim RS, Aloe AM, Becker BJ. Extracting the Variance Inflation Factor and Othe Multicollinearity Diagnostics fromTypical Regression Results. Basic Appl Soc Psych. 2017:39(2):81-90. doi: 10.1080/01973533.2016.1277529. 